\title{
ESTILO DE VIDA DE PROFESSORES DE COLÉGIOS MILITARES DO BRASIL
}

ESTILO DE VIDA DE PROFESORES DE COLEGIOS MILITARES DE BRASIL

LIFESTYLE OF TEACHERS OF MILITARY SCHOOLS OF BRAZIL.

\author{
Cati Reckelberg AZAMBUJA ${ }^{1}$ \\ Liliani Mathias BRUM ${ }^{2}$ \\ Kelly Christine Maccarini PANDOLFO ${ }^{3}$ \\ Daniella Araújo CERATTI ${ }^{4}$ \\ Daniela Lopes SANTOS 5 \\ Maria Rosa Chitolina SCHETINGER ${ }^{6}$
}

RESUMO: A prática regular de exercícios físicos, a manutenção de atividades ocupacionais e momentos de lazer ativos contribuem na saúde de cada indivíduo. Este estudo objetivou identificar o estilo de vida e o nível de atividade física dos professores de Colégios Militares do Brasil. Participaram da pesquisa 33 professores do Sistema Colégio Militar do Brasil. Na avaliação do estilo de vida utilizou o questionário "Estilo de Vida Fantástico" classificando os professores em cinco categorias. Já o nível de atividade física foi determinado por meio do "Questionário de Atividade Física Habitual de Baecke", cuja pontuação obtida classificou os professores em quatro grupos. Os participantes da pesquisa também responderam a questões sociodemográficas. Os dados foram analisados através de estatística descritiva ocorrendo posterior classificação em categorias. Os resultados demonstraram que 75,6\% dos professores apresentavam idade entre 31 e 50 anos. Na classificação do Estilo de Vida, a maioria dos professores está em condições "regular" ou "pouco ativos" quanto ao nível de atividade física. Neste sentido, conclui-se que os professores dos Colégios Militares do Brasil, de forma geral, parecem não apresentarem um estilo de vida e um nível de atividade física satisfatórios.

PALAVRAS-CHAVE: Estilo de vida. Nível de atividade física. Professores. Colégios militares.

RESUMEN: La práctica regular de actividades físicas, el mantenimiento de actividades ocupacionales y momentos de ocio activos contribuyen a la salud de cada individuo. Este estudio tiene el objetivo de identificar el estilo de vida y el nivel de

\footnotetext{
${ }^{1}$ Doutora em Educação em Ciências: Química da Vida e Saúde. UFRGS - Universidade Federal do Rio Grande do Sul. Email: cati.razambuja@ hotmail.com

${ }^{2}$ Coordenadora do Serviço de Perícia Médica. UFSM - Universidade Federal de Santa Maria. Email: lilianibrum@gmail.com

3 Mestre em Educação Física. UFSM - Universidade Federal de Santa Maria. Email: kellypandolfo@hotmail.com

4 Licenciada em Educação Física. UFSM - Universidade Federal de Santa Maria. Email: dani_ceratti@hotmail.com

5 Docente Associada do Departamento de Métodos e Técnicas Desportivas. UFSM - Universidade Federal de Santa Maria. Email: lopesdossantosdaniela@gmail.com

${ }^{6}$ Docente Associada do Departamento de Química. UFSM - Universidade Federal de Santa Maria. Email: mariachitolina@gmail.com
} 
actividad física de los profesores de Colegios Militares de Brasil. Participaron de esta investigación 33 profesores del Sistema Colegio Militar de Brasil. En la evaluación del estilo de vida se utilizó el cuestionario "Estilo de Vida Fantástico", clasificando los profesores en cinco categorías. Respecto al nivel de actividad física fue determinado por medio del "Cuestionario de Actividad Física Habitual de Baecke", cuya puntuación obtenida pudo clasificar los profesores en cuatro grupos. Los participantes de la investigación también contestaron a preguntas sociodemográficas. Los datos fueron analizados por medio de estadística descriptiva con posterior clasificación en categorías. Los resultados demostraron que el 75,6\% de los profesores presentaban edad entre 31 y 50 años. En la clasificación del Estilo de Vida, la mayoría de los profesores está en condiciones "regular" y "poco activos" con relación al nivel de actividad física. En este sentido, se concluye que los profesores de los Colegios Militares de Brasil, de forma general, parecen no presentar un estilo de vida y un nivel de actividad física satisfactorios.

PALABRAS CLAVE: Estilo de vida. Nivel de actividad física. Profesores. Colegios militares.

ABSTRACT: The regular practice of physical exercise, maintaining occupational and leisure time contribute assets in the health of each individual. This study aimed to identify the lifestyle and level of physical activity of teachers Military Colleges of Brazil. Participants were 33 teachers Military College System of Brazil. In evaluating the lifestyle questionnaire used the "Fantastic Lifestyle" classifying teachers into five categories. Already, the level of physical activity was determined using the "Questionnaire of Baecke Habitual Physical Activity" whose scores qualified teachers into four groups. Survey participants also answered questions sociodemographic. Data were analyzed using descriptive statistics occurring subsequent categorization. The results showed that $75.6 \%$ of teachers were aged between 31 and 50 years. In the classification of Lifestyles, most teachers are in condition "regular" and "somewhat active" in the level of physical activity. In this sense, it is concluded that teachers of Military Colleges of Brazil, in general, do not seem to present a lifestyle and a satisfactory level of physical activity.

KEYWORDS: Lifestyle. Physical activity. Teachers. Military schools.

\section{Introdução}

A Educação Brasileira reflete as consequências dos movimentos de globalização que, no decorrer das últimas décadas, vem gerando profundas e complexas transformações, desde a estrutura organizacional das Instituições de Ensino e de seus objetivos de educação, até a atuação dos professores (MATOS; SARDINHA, 1999) que inclui, segundo Coutinho et al. (2012), o desenvolvimento da capacidade dos alunos para atuarem como cidadãos ativos na sociedade. 
Entre estas instituições de ensino, o Sistema Colégio Militar do Brasil (SCMB) é formado por 12 unidades distribuídas em todas as regiões do país e oferece turmas para alunos concursados e amparados por Lei, do $6^{\circ}$ Ano do Ensino Fundamental até o $3^{\circ}$ Ano do Ensino Médio, propiciando educação de alta qualidade para, aproximadamente, 15 mil jovens (BRASIL, 2010).

Diante desta necessária reorganização das escolas, estas podem ser consideradas como espaços privilegiados, pois unem professores e alunos em um mesmo ambiente que pode/deve cumprir vários papéis. Entre eles, pode-se destacar a influência exercida pelos professores sobre os alunos, através de exemplos de conduta, assim como-, tornar possível que os adolescentes reconheçam o valor da saúde (GINZBERG, 1991).

De acordo com a Lei de Diretrizes e Bases da Educação Nacional (LDBEN), todos os estabelecimentos de ensino do País devem possuir uma proposta pedagógica própria, verdadeira, síntese dos objetivos e da orientação que imprimem à ação educacional (BRASIL, 1996). Entre outras características, a proposta pedagógica dos Colégios Militares prioriza princípios e práticas de um ensino moderno e atual que incluem a criação de hábitos saudáveis para o corpo (BRASIL, 1999).

Os determinantes do comportamento de risco e estilo de vida dos professores integrados no sistema educativo tornam-se condição necessária para o desenvolvimento de estratégias preventivas adequadas a este grupo, sendo que pesquisas populacionais que forneçam subsídios na análise do estilo de vida dos educadores têm sido desenvolvidas em estudos que procuram estabelecer a proporcionalidade entre saúde e bem-estar com a melhora do estilo de vida (MATSUDO et al., 2002).

\section{Saúde e fatores de risco}

A saúde deve ser vista como objeto de intervenção que torna possível a melhora do estilo de vida do indivíduo e, as ações, devem ser direcionadas às condições de vida e a fatores sociais, já que estes interagem para manter os padrões de comportamento (RODRIGUEZ-AÑEZ; REIS; PETROSKI, 2008).

Sendo assim, a qualidade de vida (SHEPHARD, 1996), o comportamento e o estilo de vida são determinantes cruciais para a saúde de um indivíduo (MADUREIRA; FONSECA; MAIA, 2003), tornando-se importante analisar os processos que estão envolvidos na escolha dos estilos de vida (WHO, 1998). Além disso, ao observar sobre a questão laboral, consta na literatura que baixos índices de bem-estar no trabalho 
podem provocar consequências, tanto para o indivíduo quanto nas organizações sociais e instituições (FERNANDES et al., 2009).

Se for analisada a história, a segunda metade do séc. XX é marcada pelo surgimento da "Era das Doenças Crônico-Degenerativas" com seu paradigma predominante "caixa-preta", o qual relaciona exposição ao efeito, ou seja, uma rede multicausal de fatores determinantes da doença. Entre os principais determinantes estão: o estilo de vida, o meio ambiente e os aspectos sociais (SUSSER; SUSSER, 1996).

Estes determinantes possuem vários elementos ocultos de agravo à saúde, como por exemplo, o sedentarismo. Os modelos multicausais na determinação de agravos possuem três teorias: 1) Teoria do germe, causadora de doenças infecciosas; 2) Teoria ambiental, causadora de doenças pela poluição no meio ambiente; 3) Teoria do estilo de vida, causadora de doenças por causas comportamentais refletidas pelo estresse, sedentarismo, ingestão de álcool, tabagismo e alimentação inadequada. Ainda, segundo o autor, a atividade física relacionada à saúde (diferente da relacionada à aptidão) aparece como fator modificador no risco de desenvolvimento de doenças crônicodegenerativas (PITANGA, 2002).

Apesar da evolução conceitual a respeito da saúde levar em consideração o estado de saúde positiva ou bem-estar, até bem pouco tempo atrás, para avaliação de estudos epidemiológicos, os indicadores de saúde eram somente negativos, como a taxa de mortalidade e morbidade, e em muitas vezes tomava-se como base do nível de saúde de determinada localidade apenas a taxa de mortalidade infantil (NAHAS, 1996).

Com o surgimento dessas novas ideias, passaram também a ser considerados os indicadores positivos, levando-se em conta outros determinantes para alcançar a almejada qualidade de vida, tais como: orgânicos ou biológicos (saúde e doença), psicológicos (identidade, autoestima, criatividade, habilidade), sociais (vida familiar, vida sexual, relacionamentos), comportamentais (vida profissional, hábitos, repouso, lazer), materiais (habitação, bens, renda) e estruturais (concepção sociopolítica, posição social) (TOLEDO, 2006). Portanto, a saúde não é uma condição estática, existente somente devido à chamada ausência de doenças, mas sim um processo de aprendizagem, tomada de decisão e ação para a otimização do bem-estar próprio.

Está provado que vários fatores de risco de doenças crônicas e cardiovasculares estão presentes ou se iniciam já no período da infância. Estes fatores estão relacionados aos hábitos de vida, como o exercício físico, o tabagismo e a dieta (PIÉRON, 2004). No Brasil, estima-se que as doenças cardiovasculares (DCV) respondam por 
aproximadamente 350 mil dos óbitos ocorridos entre a população por causas conhecidas, representando $29 \%$ e liderando as causas de mortalidade (SOCIEDADE BRASILEIRA DE CARDIOLOGIA, 2016). Portanto, aqueles jovens que eventualmente venham a apresentar fatores de risco, com o avanço da idade tendem a apresentar maior predisposição ao aparecimento de processos aterosclerótico e consequente desenvolvimento das DCV (GUEDES et al., 2006).

As características dos fatores de risco predisponentes às DCV são conhecidas como de natureza biológica (quantidade de gordura corporal, do perfil lipídicolipoprotéico plasmático e níveis de pressão arterial) e de natureza comportamental (prática de atividade física, hábitos alimentares e uso de tabaco), pois segundo Guedes et al. (2006), em se tratando de sujeitos jovens, momento em que são adquiridos e incorporados comportamentos de difícil modificação em idades futuras, torna-se prudente analisar não apenas os fatores de risco biológicos de forma isolada, mas também aqueles de natureza comportamental.

Estas constatações confirmam que todos os esforços para tentar detectar precocemente a presença de fatores de risco no jovem e no adulto jovem possibilitam planejamento e implantação de programas intervencionistas preventivos direcionados à redução da probabilidade de manifestação das DCV futuramente na idade adulta.

\section{Estilo de vida e atividade física habitual}

O estilo de vida é caracterizado por padrões de comportamento identificáveis que podem ter um efeito profundo na saúde dos seres humanos e está relacionado com diversos aspectos que refletem as atitudes, os valores e oportunidades na vida das pessoas (WHO, 1998). O conceito de estilo de vida saudável adquiriu importância a partir de 1990 e está associado a hábitos de atividade física regular, entre outros cuidados com a saúde, relacionando-se a maior qualidade de vida geral, bem-estar físico e mental (RICHARDSON et al., 2005; TELEMA; NUPPONEN; PIÉRON, 2005).

O estilo de vida dos professores, considerando-se que o exercício do magistério é uma função antiga e desgastante, sofre as consequências dos problemas decorrentes dela desde os primórdios. Alguns estudos brasileiros (BRUM et al., 2012; FERNANDES et al., 2009; VIEIRA, 2007; GASPARINI; BARRETO; ASSUNÇÃO, 2005) demonstram uma relação importante entre a saúde dos professores e suas condições de vida e de trabalho, demonstrando que muitos docentes têm estilos de vida 
precários com relativa presença de atividade física, poucas horas de sono durante a noite e muitos problemas de saúde, destacando os transtornos músculos esqueléticos, respiratórios e mentais. Além disso, vários fatores de riscos podem fazer parte da rotina, entre eles o ruído, uso constante da voz, movimentos repetitivos, trabalho estressante, cansativo e desgastante tanto mental como fisicamente (VEDOVATO; MONTEIRO, 2008).

A contribuição da atividade física para a saúde está associada a uma diminuição do nível de risco ao qual cada pessoa está sujeita durante a vida (PIÉRON, 2004). A pesquisa sobre estilo de vida e a participação em atividades físicas e esportistas mostrou que as percepções dos participantes sobre sua saúde, capacidades atléticas e aspecto físico exerciam influências que frequentemente foram determinantes (DISHMAN, 1990; DUDA, 1989). Segundo dados do World Health Organization (1998), a prática regular de atividade física beneficia a preservação da saúde, porque reduz os riscos de adoecimento por diabetes, hipertensão e problemas cardiovasculares, além de prevenir as dores musculoesqueléticas promovendo o bem-estar psicológico, reduzindo o estresse, a ansiedade e a depressão.

\section{Sistema de ensino no exército brasileiro}

O ensino oferecido pelo Exército Brasileiro (EB) é composto por duas etapas: a) Preparatória, destinada ao ingresso na Escola Preparatória de Cadetes do Exército EsPCEx e escolas de nível superior das Forças Armadas; b) Assistencial, destinado aos anos finais do Ensino Fundamental e aos anos do Ensino Médio ofertado pelo SCMB. Os Colégios Militares que compõem o SCMB são regidos pelas Normas para Planejamento e Conduta do Ensino (NPCE) do EB e, segundo o Regulamento dos Colégios Militares (R/69), instrumento regulador do ensino nestas instituições, a educação segue a Legislação Federal concomitantemente às leis e regulamentos em vigor no EB (BRASIL, 2008).

O SCMB foi instituído em 07 de fevereiro de 1973 como resultado da criação da Diretoria de Ensino Preparatório e Assistencial (DEPA). Porém, o histórico dos colégios militares, no Brasil, tem início com a Resolução Imperial de 09 de março de 1889, onde Tomás José Coelho de Almeida, após ter assumido o Ministério da Guerra, atendeu ao antigo pedido dos militares, através do Decreto Imperial n 10.202 , criando o Colégio Militar da Corte, que ficou conhecido por Imperial Colégio Militar. Segundo Gil (2003), 
no Brasil, em 1912 já havia três Colégios Militares: Rio de Janeiro, Porto Alegre (criado pelo Decreto $\mathrm{n}^{\circ}$ 9.397, de 28 de fevereiro de 1912) e Barbacena (criado pelo Decreto $\mathrm{n}^{\circ}$ 9.507, de 03 de abril do mesmo ano), o qual foi extinto em 1925. Seguiram-se os Colégios Militares de Fortaleza (1919), Belo Horizonte (1955), Salvador (1957), Curitiba (1958), Recife (1959), Manaus (1971), Brasília (1978), Campo Grande e Juiz de Fora (1993) e Santa Maria (1994).

As metas gerais da proposta pedagógica do SCMB são: a) Permitir ao aluno desenvolver atitudes e incorporar valores familiares, sociais e patrióticos que lhe assegurem um futuro cidadão patriota, cônscio de seus deveres, direitos e responsabilidades, qualquer que seja o campo profissional de sua preferência; b) propiciar ao aluno a busca e a pesquisa continuada de informações relevantes; c) desenvolver no aluno a visão crítica dos fenômenos políticos, econômicos, históricos, sociais e científico-tecnológicos, ensinando-os, pois, a aprender para a vida e não mais simplesmente para fazer provas; d) preparar o aluno para refletir e compreender os fenômenos e não meramente memorizá-los; e) capacitar o aluno à absorção de prérequisitos fundamentais ao prosseguimento dos estudos acadêmicos e não de conhecimentos supérfluos que se encerram em si mesmos; f) estimular o aluno para a saudável prática da atividade física, buscando o seu desenvolvimento físico e incentivando a prática habitual do esporte; g) despertar vocações para a carreira militar (BRASIL, 2008).

Os professores dos Colégios Militares possuem diversas atribuições pedagógicas e militares. Entre tantas responsabilidades encontram-se a participação nos trabalhos pedagógicos previstos de recuperação, integração às bancas examinadoras, participação ativa na execução da proposta pedagógica do colégio militar, elaborar documentos e participar da Avaliação Diagnóstica, quando designado. Além disso, o R/69 e o Regimento Interno dos Colégios Militares (RI/CM) descrevem extensa lista de atribuições (BRASIL, 2009; BRASIL, 2008).

Diante disso, a motivação principal deste estudo foi identificar o estilo de vida e o nível de atividade física dos professores dos Colégios Militares do Brasil, onde a pesquisa proposta buscou fornecer subsídios para um acompanhamento, desenvolvimento de estratégias e direcionamento de ações preventivas e educativas relativas aos comportamentos que compõem o estilo de vida dos docentes. 


\section{Métodos}

O presente estudo foi aprovado pelo Comitê de Ética em Pesquisa com Seres Humanos da Universidade Federal de Santa Maria, reconhecida pela Comissão Nacional de Ética em Pesquisa (CONEP) do Conselho Nacional de Saúde (CNS), sob o protocolo de Certificado de Apresentação para Apreciação Ética (CAAE) n. ${ }^{\circ}$ 0094.0.243.000-11. Os voluntários, depois de esclarecidos sobre a proposta do estudo e os procedimentos aos quais seriam submetidos, assinaram o Termo de Consentimento Livre e Esclarecido (TCLE).

Participaram do estudo 33 professores, de ambos os sexos, do SCMB. Destes, 20 eram do sexo masculino e 13 do sexo feminino. Como critério de inclusão selecionou-se aqueles que atuavam como docentes em colégios militares há pelo menos três meses.

Os participantes da pesquisa responderam a questões sociodemográficas como idade, sexo, estado civil, nível de escolaridade, jornada de trabalho docente e estado geral de saúde. Além disso, também foi solicitado que cada indivíduo registrasse seu peso e estatura auto referidos (SILVEIRA et al., 2005) para posterior cálculo do Índice de Massa Corpórea (IMC).

Para calcular o IMC, foi utilizado o índice de Quetelet, expressão que divide a massa corporal pela estatura elevada ao quadrado. Segundo a classificação da WHO (2006), indivíduos com IMC ( $\left.\mathrm{kg} / \mathrm{m}^{2}\right)$ : a) menor que 18,5 (baixo peso); b) 18,5 a 24,9 (peso adequado); c) 25 a 29,9 (pré-obeso); d) 30 a 34,9 (obesidade classe I); e) 35 a 39,9 (obesidade classe II); f) igual ou maior que 40 (obesidade classe III).

Para avaliar o estilo de vida dos docentes utilizou-se o "Questionário Estilo de Vida Fantástico" (QEVF) proposto pela Sociedade Canadense de Fisiologia do Exercício em 1998, traduzido e validado para o Brasil (RODRIGUEZ-AÑEZ; REIS; PETROSKI, 2008). O questionário possui vinte e cinco questões divididas em nove domínios: 1) Família e amigos; 2) Atividade Física; 3) Nutrição; 4) Tabaco e tóxicos; 5) Álcool; 6) Sono, cinto de segurança, estresse e sexo seguro; 7) Tipo de comportamento; 8) Introspecção; 9) Trabalho. O QEVF é um instrumento autoaplicado que permite determinar a associação entre o estilo de vida e a saúde através de escala do tipo Likert pontuadas conforme o valor de cada coluna, sendo: 0 (zero) para a primeira coluna; 1 (um) para a segunda coluna; 2 (dois) para a terceira coluna; 3 (três) para a quarta coluna; 4 (quatro) para a quinta coluna. Para questões binárias, a pontuação é 0 (zero) para a primeira e 4 (quatro) para a última coluna. Ao final do questionário, a soma de 
todas as pontuações permite que os professores sejam classificados em cinco categorias: a) Excelente (85 a 100 pontos); b) Muito Bom (70 a 84 pontos); c) Bom (55 a 69 pontos); d) Regular (35 a 54 pontos); e) Necessita Melhorar (0 a 34 pontos).

Para determinação do nível de atividade física foi utilizado o "Questionário de Atividade Física Habitual” (QAFH) elaborado por Baecke; Burema; Frijters (1982), traduzido e adaptado para população brasileira por Sardinha et al. (2010). A pontuação obtida no QAFH permite classificar os professores em quatro grupos: a) Inativos (0 a 5 pontos); b) Pouco ativo (6 a 11 pontos); c) Ativo (12 a 20 pontos); d) Muito ativo (21 ou mais pontos). Os resultados foram analisados através de estatística descritiva, representada por média e desvio padrão para a pontuação do questionário e posteriormente classificados em categorias.

\section{Resultados e discussão}

A amostra do estudo foi composta por 33 docentes de Colégios Militares do Brasil, sendo 60,6\% ( $n=20)$ do sexo masculino e 39,4\% $(n=13)$ do sexo feminino. A prevalência do sexo masculino encontra-se em discordância com trabalhos que investigaram professores de escolas não pertencentes ao SCMB (BRUM et al., 2012; GASPARINI; BARRETO; ASSUNÇÃO, 2005); Segundo Fernandes et al. (2009), culturalmente, existe uma tendência de relacionar a atividade ao "papel feminino" de cuidar e educar.

A faixa etária de 75,8\% (n=25) dos professores encontrava-se compreendida entre o intervalo de 31 e 50 anos, o que pode ser justificado pelo fato de que os Colégios Militares que integram o SCMB realizam criteriosas seleções para o seu quadro docente, exigindo de seus professores qualificação e formação continuada, o que só pode ser atingido com alguns anos de estudo. Já, o estado nutricional, verificado por meio do cálculo do IMC, apontou que tanto homens ( $\mathrm{IMC}=26,0 \pm 2,5 \mathrm{~kg} / \mathrm{m}^{2}$ ) quanto mulheres $\left(\mathrm{IMC}=27,1 \pm 3,4 \mathrm{~kg} / \mathrm{m}^{2}\right)$ encontravam-se acima do peso, sendo classificados como pré-obesos (ou sobrepeso). Mesmo o IMC sendo uma medida frágil para avaliar partes específicas do corpo, tem sido utilizado em estudos epidemiológicos para indicar o sobrepeso (DAMASCENO et al., 2005).

Com relação ao estado civil, $63,6 \%$ dos docentes $(n=21)$ responderam que são casados, seguidos de 24,2\% $(n=8)$ que afirmaram estar solteiros. A grande maioria $(78,8 \% ; n=26)$ que aceitou participar da pesquisa reside nos estados das regiões Sudeste 
$(51,5 \% ; n=17)$ e Sul $(27,3 \% ; n=9)$. Quanto à formação acadêmica dos professores, 24,2\% ( $n=8)$ formaram-se em cursos da área de Ciências (biologia, física, química e matemática), seguidos daqueles que se licenciaram em Educação Física (18,2\%; n=6) e daqueles que colaram grau em Pedagogia e Letras (Português/Línguas) (18,2\%; n=6). Boa parte dos docentes possui pós-graduação, sendo 45,5\% (n=15) especialização e 33,3\% (n=11) mestrado acadêmico.

Os professores do SCMB podem ser de duas naturezas: militares de carreira e civis. Neste estudo, $57,6 \%(n=19)$ são militares e $42,4 \% \quad(n=14)$ são civis. Os professores civis, assim como os militares, encontram-se sob regência das normas da instituição. Neste sentido, Vieira (2007) entende que as normas, regras, determinações e leis que guiam os professores civis e militares são as mesmas, entretanto o professor militar também deve cumprir os preceitos militares inerentes à função militar.

Outra característica verificada na amostra foi o tempo de docência no SCMB que, para 36,4\% ( $\mathrm{n}=12)$ dos professores, encontra-se entre 11 e 20 anos. Ainda, foi constatado que 78,8\% ( $\mathrm{n}=26)$ destes docentes lecionaram em apenas um dos Colégios Militares existentes, apesar de boa parte deles serem militares de carreira e estarem sujeitos às transferências entre as Unidades de Ensino espalhadas pelo território nacional.

A jornada de trabalho semanal de 48,5\% $(n=16)$ dos professores encontra-se compreendida entre 31 e 40 horas, sendo que o número de horas lecionando em sala de aula- para 69,7\% (n=23) deles está entre 11 e 20 horas. Estes dados demonstram a diferença de carga horária da maioria dos professores quando comparados aos de escolas públicas, os quais quando questionados afirmaram lecionar de trinta a quarenta horas semanais (85\%) e $71 \%$ afirmaram que o faziam em duas escolas (BRUM et al., 2012). A diferença encontrada entre a jornada semanal total e o número de horas em sala de aula é explicada por Vieira (2007) ao afirmar que os professores realizam atividades paralelas às obrigações da função docente, tais como: escalas de representações, chamadas fora do horário de expediente diurno de trabalho, preparação de aulas e atividades, reuniões e atendimento aos alunos.

Os professores dos Colégios Militares possuem diversas atribuições pedagógicas e militares. Entre tantas responsabilidades encontram-se a participação nos trabalhos pedagógicos previstos de recuperação, integração às bancas examinadoras, participação ativa na execução da proposta pedagógica do Colégio Militar, elaborar documentos e participar da Avaliação Diagnóstica, quando designado. Além disso, o R/69 e o 
Regimento Interno dos Colégios Militares (RI/CM) descrevem extensa lista de atribuições (BRASIL, 2010; BRASIL, 2008).

Quando perguntados sobre seu estado geral de saúde, 21,2\% (n=7) dos docentes responderam possuir algum tipo de doença diagnosticada. Entre elas, foram citadas, hipertensão arterial sistêmica, diabetes, dislipidemia e obesidade. O desenvolvimento das doenças crônicas não transmissíveis (DCNT) pode ser reduzido com a adoção de hábitos de vida saudáveis (ACSM, 1996). Segundo autores como Nahas (2003) e Shephard (1996), um estilo de vida considerado saudável inclui as diversas dimensões que abrangem o cotidiano das pessoas, tais como nutrição balanceada, atividade física, comportamento preventivo, relacionamento social e controle do estresse.

“Apesar de todas as evidências no que se refere às atitudes que promovam um estilo de vida saudável e aos efeitos dele decorrentes, as pessoas, de modo geral, possuem hábitos de vida bastante diferentes do que pode ser considerado ideal" (COSTA; MILANI, 2007). Diante desta afirmação, nota-se que existe uma dificuldade natural das pessoas realizarem na prática os conceitos e teorias sobre comportamentos saudáveis.

O período de horas de sono apresenta relação com o aumento do índice de massa corpórea (PATEL et al., 2006; KOHATSU et al., 2006; KNUTSON, 2005), sendo que indivíduos com idade entre 32 e 49 anos com tempo de sono menor de sete horas apresentam índice de massa corpórea próxima da obesidade (GANGWISCH et al., 2005). Entre os participantes da pesquisa, 54,6\% $(n=18)$ dos professores afirmaram dormir de cinco a seis horas durante os dias de semana, enquanto que, 39,4\% ( $n=13)$ disseram dormir de sete a oito horas. Já nos finais de semana, 69,7\% (n=23) dos entrevistados responderam que dormem mais de oito horas por noite. Estes dados, juntamente com a classificação de sobrepeso dos professores da amostra, encontram-se em conformidade com os autores acima citados. Ainda relacionado ao assunto, 36,4 $(\mathrm{n}=12)$ responderam que tem o hábito de realizar quatro refeições diárias (café da manhã, almoço, lanche e janta).

$\mathrm{Na}$ classificação do Estilo de Vida, 9,1\% (n=3) dos professores estão em condição "Regular", 45,6\% (n=15) enquadraram-se na categoria "Bom", 39,4\% (n=13) em "Muito Bom" e somente 6,1\% $(\mathrm{n}=2)$, como "Excelente". No estudo realizado com os docentes da Universidade Estadual do Sudoeste da Bahia, a aplicação do mesmo instrumento verificou que 55,26\% possuíam "Bom" e 44,74\% "Excelente" estilo de vida. Os professores universitários da Bahia apresentaram, de forma geral, melhores 
classificações do que os que lecionam no SCMB. Tais resultados sugerem que analisar os processos responsáveis pelas escolhas que levam a um determinado estilo de vida é fundamental e este se torna decisivo para a saúde de um indivíduo (FERNANDES et al., 2009).

Quando estes dados foram analisados na sua totalidade e/ou estratificados por sexo, em termos de média e desvio padrão, verificou-se que a pontuação manteve-se dentro da mesma classificação para os dois sexos, sugerindo que a atividade docente militar apesar de exigir diversas atribuições destes profissionais não chega a afetar negativamente no estilo de vida destes profissionais (Tabela 1).

Tabela 1 - Pontuação obtida pelos Docentes do Sistema Colégio Militar do Brasil no Questionário Estilo de Vida Fantástico, estratificada por sexo.

\begin{tabular}{|c|c|c|c|}
\hline \multicolumn{4}{|c|}{ ESTILO DE VIDA FANTÁSTICO } \\
\hline & & Pontuação (X/DP) & Classificação \\
\hline Masculino & $(\mathbf{n}=20)$ & $68,3 / 13,8$ & Bom \\
\hline Feminino & $(n=13)$ & $67,3 / 9,04$ & Bom \\
\hline
\end{tabular}

Fonte: Os Autores com base nos dados coletados na pesquisa.

$\mathrm{X}$ : média; DP: desvio padrão.

A comparação do EV dos professores do SCMB medido e percebido apontou que $78,13 \%(\mathrm{n}=25)$ foram avaliados pelo instrumento como detentores de um "Bom", "Muito Bom" ou "Excelente" EV, enquanto que, ao serem questionados sobre como classificariam seu EV, 90,62\% (n=30), perceberam seu EV desta maneira. A superestimação nesta avaliação pode ter ocorrido pela diferença na importância que cada domínio do EV exerce sobre o indivíduo. Outro dado importante foi o de que 18,75\% ( $\mathrm{n}=6$; sendo três homens e três mulheres) dos docentes foram incluídos na classificação de "Necessita Melhorar", apesar de nenhum deles ter percebido seu EV desta forma.

Pode-se observar que as mulheres apresentaram a mesma classificação do que os homens. Tanto o grupo masculino quanto o feminino pontuaram no limiar superior da categoria "Bom", segundo a classificação deste instrumento. A literatura não apresenta estudos sobre o estilo de vida de professores, especificamente os de Colégios Militares, dificultando a comparação dos resultados da presente pesquisa.

Segundo a WHO (1998), o estilo de vida é caracterizado por padrões de comportamento identificáveis que podem ter um efeito profundo na saúde dos seres 
humanos e está relacionado com diversos aspectos que refletem as atitudes, os valores e as oportunidades na vida das pessoas. Sendo assim, podemos afirmar que o comportamento adotado pelas pessoas, assim como o estilo de vida, são determinantes cruciais para a saúde das mesmas, assim, com os resultados encontrados neste estudo, podemos vislumbrar que a maioria destes profissionais pode estar gozando de uma boa saúde.

O instrumento utilizado para avaliar o estilo de vida dos professores é composto por nove domínios que possuem diferentes pesos na classificação do perfil desta variável. Dos pontos possíveis de serem somados, $8 \%$ (8 pontos) são de questões referentes à Família e Amigos; 8\% (8 pontos) para Atividade Física; 12\% (12 pontos) para Nutrição; 12\% (12 pontos) para uso de Tabaco e Tóxicos; 16\% (16 pontos) para uso de Bebidas Estimulantes e Álcool; 20\% (20 pontos) para Segurança e Cuidados Próprios; 4\% (4 pontos) para Comportamento; 16\% (16 pontos) para a Introspecção; 4\% (4 pontos) para o Trabalho. A Tabela 2 apresenta os dados colhidos entre os professores.

Tabela 2 - Pontuação obtida pelos Docentes do Sistema Colégio Militar do Brasil nos domínios no Questionário Estilo de Vida Fantástico.

\begin{tabular}{cccc}
\hline & \multirow{2}{*}{ Pontos } & \multicolumn{2}{c}{ Pontos Alcançados } \\
\cline { 3 - 4 } & Possíveis & $\mathbf{X}$ & DP \\
\hline Família e Amigos & 8 & 5,9 & 1,9 \\
Atividade Física & 8 & 4,1 & 2,5 \\
Nutrição & 12 & 7,1 & 4,2 \\
Tabaco e Tóxicos & 12 & 10,5 & 2,5 \\
Bebidas Estimulantes e Álcool & 16 & 11,8 & 4,2 \\
Segurança e Cuidados Próprios & 20 & 13,4 & 5,1 \\
Comportamento & 4 & 1,6 & 1,3 \\
Introspecção & 16 & 10,8 & 3,8 \\
Trabalho & 4 & 2,7 & 1,1 \\
Total & $\mathbf{1 0 0}$ & $\mathbf{6 7 , 9}$ & - \\
\hline
\end{tabular}

Fonte: Os Autores com base nos dados coletados na pesquisa. $\mathrm{X}$ : média; DP: desvio padrão.

Analisando a composição do instrumento que avalia o estilo de vida, percebe-se que apenas três domínios (segurança e cuidados próprios; bebidas estimulantes e álcool; introspecção) são responsáveis por mais da metade (52) dos pontos possíveis, 
reafirmando a importância destes hábitos em relação à totalidade. $\mathrm{O}$ grupo avaliado somou, em média, 36 pontos (69,2\% dos pontos possíveis para estes três domínios). Outros quatro domínios (família e amigos; atividade física; nutrição; tabaco e tóxicos) são responsáveis por mais 40 pontos do questionário. Os professores que participaram deste estudo somaram, em média, 27,6 pontos (69\% dos pontos possíveis nestes quatro domínios). Finalmente, os domínios comportamento e trabalho são responsáveis por oito pontos possíveis. Neste estudo, os docentes atingiram, em média, 4,3 pontos (53,8\% dos pontos possíveis para estes dois domínios).

Diante dos dados obtidos, percebe-se que o resultado mais preocupante diz respeito aos domínios referentes ao comportamento e ao trabalho. Autores como Guimarães et al. (2006) afirmam que profissões ligadas à Educação são altamente estressantes, considerando-se as atuais estruturas das escolas e a constante modernização do ensino. A prática diária dos professores dos Colégios Militares é repleta de regras e recomendações para o bom desempenho da função. Ainda, é fundamental o desenvolvimento de características ligadas ao conceito de Hardiness, tais como: controle, desafio e comprometimento, que possibilitam a existência de professores saudáveis, com capacidade para transformar o estresse em experiências enriquecedoras e positivas.

O Nível de Atividade Física (NAF) dos docentes (Tabela 3) foi avaliado segundo o instrumento proposto por Baecke; Burema; Frijters (1982) e validado por Sardinha et al. (2010), apresentando valores total (ET) e fracionados para as atividades físicas ocupacionais (AFO), exercícios físicos (AFL) e momentos de lazer (ALL).

Tabela 3 - Pontuação obtida pelos Docentes do Sistema Colégio Militar do Brasil no Questionário Nível de Atividade Física Habitual, estratificada por sexo.

\begin{tabular}{ccccccc}
\hline \multirow{2}{*}{$\begin{array}{c}\text { Nível Atividade } \\
\text { Física }\end{array}$} & \multicolumn{2}{c}{ Masculino } & \multicolumn{2}{c}{ Feminino } & \multicolumn{2}{c}{ Total } \\
\cline { 2 - 7 } & $\mathbf{X}$ & $\mathbf{D P}$ & $\mathbf{X}$ & $\mathbf{D P}$ & $\mathbf{X}$ & DP \\
AFO & 2,74 & 0,44 & 3,09 & 0,62 & 2,88 & 0,55 \\
EFL & 5,67 & 4,03 & 2,75 & 0,67 & 2,96 & 3,83 \\
ALL & 2,18 & 0,70 & 2,00 & 0,71 & 2,11 & 0,77 \\
ET & 8,02 & 1,28 & 7,14 & 1,20 & 7,95 & 1,25 \\
\hline
\end{tabular}

Fonte: Os Autores com base nos dados coletados na pesquisa.

AFO: atividades físicas ocupacionais; AFL: exercícios físicos; ALL: momentos de lazer; ET: escore total; X: média; DP: desvio padrão. 
O Nível de Atividade Física Habitual dos professores do SCMB totalizou a média de 7,95 $\pm 1,25$ pontos, sendo que a estratificação do mesmo em três diferentes contextos (trabalho - AFO; exercícios físicos - EFL; momentos de lazer - ALL) obteve

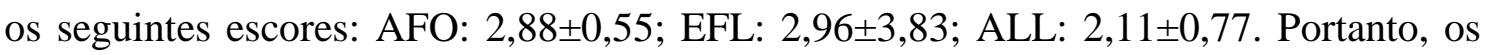
docentes deste estudo foram classificados como "Pouco Ativos". A literatura não apresenta estudos sobre o nível de atividade física de professores de Colégios Militares, dificultando a comparação dos resultados da presente pesquisa.

Foi analisado o NAF dos professores do SCMB medido e percebido. A maioria dos docentes $(68,75 \% ; \mathrm{n}=22)$ percebe-se como "Ativo" ou "Muito Ativo", sendo esta uma condição mais prevalente entre os professores do sexo masculino. Notou-se que há uma avaliação superestimada no momento da percepção, o que pode indicar uma distorção em relação à quantificação das atividades desenvolvidas normalmente. Contudo, é importante registrar que $28,13 \%(n=9)$ tiveram sua percepção confirmada com a aplicação do instrumento que mede o NAF.

A contribuição da atividade física para a saúde está associada à diminuição do nível de risco ao qual cada pessoa está sujeita durante a vida. Além disso, a prática regular de exercícios físicos e a manutenção de atividades ocupacionais e momentos de lazer ativos colaboram para a prevenção de doenças crônico-degenerativas, dores musculoesqueléticas e promoção do bem-estar psicológico por meio da redução do estresse, da ansiedade e da depressão (ARAÚJO; ARAÚJO, 2000).

Segundo Corbin; Lindsey (1994), a participação regular e frequente em atividades físicas e esportivas, mesmo que sejam muito diversas, constitui um elemento chave que permite obter efeitos benéficos para a condição física e a saúde. No estudo realizado por Vedovato; Monteiro (2008), os autores verificaram que 43,4\% dos professores estudados encontravam-se sedentários e com grande quantidade de doenças com diagnóstico médico $(82,3 \%)$.

Estudos têm demonstrado que a atividade física promove importante impacto nas dimensões que compõem o estilo de vida. Sendo assim, a participação em programas de atividades física possui vários benefícios, desde os fisiológicos até os psicológicos positivos, como a melhora do humor, redução do estresse e aumento da autoestima (VALOIS et al., 2008; MORENO; CERVILLÓ; MORENO, 2008). Contudo, segundo Piéron (2004), a pouca frequência na participação não cumpre as recomendações mínimas para que haja efeitos ligados à saúde. Em outro estudo realizado por este grupo de pesquisa com professores da área de ciências de uma escola 
pública do Rio Grande do Sul, foi verificado que 28,57\% dos professores praticavam exercícios físicos cinco vezes por semana (BRUM et al., 2012). Vedovato; Monteiro (2008) encontraram em sua pesquisa que $56,6 \%$ dos professores paulistas praticavam atividade física regularmente pelo menos três vezes na semana.

\section{Considerações finais}

A interação de hábitos alimentares adequados com a realização de atividades físicas regulares frente ao desenvolvimento das atividades profissionais é um campo complexo de estudo que abrange a área de Ciências da Saúde. O grupo de professores dos Colégios Militares do Brasil apresentou estilo de vida bom, porém os homens apresentaram nível mais elevado quando comparados com o das mulheres, fato que se entende estar relacionado com a organização e disciplina militar colaborando positivamente na vida desses professores do sexo masculino. Há de se ressaltar que, individualmente, quase a metade dos docentes foi classificada com estilo de vida regular, fato este que remete a condições de vida pontuais e que merecem mais atenção.

A análise dos resultados desse trabalho permite repensar modificações nos aspectos que abrangem o desenvolvimento das atividades físicas dos professores dos Colégios estudados, pois é sabido que o estímulo para a realização da prática regular de exercícios físicos é uma conduta incentivada e avaliada periodicamente por meio de Testes de Aptidão Física, porém os professores apresentaram atividade física aquém do esperado. Destacamos a necessidade de mais estudos sobre a relação entre os diversos domínios que compõem o estilo de vida dos docentes da educação básica militar, visto que muitos destes comportamentos assumidos pelos professores podem ser repassados e copiados pelos alunos. Por fim, sugerimos ainda que este tipo de pesquisa seja estendido a outras escolas que possuem a mesma concepção de educação e exigência para seus professores.

\section{REFERÊNCIAS}

AMERICAN COLLEGE OF SPORTS MEDICINE (ACSM). Teste de esforço e prescrição de exercício. Rio de Janeiro: Revinter, 1996.

ARAÚJO, D. S. M. S.; ARAÚJO, C. G. S. Aptidão física, saúde e qualidade de vida relacionada à saúde em adultos. Rev Bras Med Esporte, v.6, n.5, p.194-203, 2000. 
BAECKE, J. A. H.; BUREMA, J.; FRIJTERS, J. E. R. A short questionnaire for the measurement of habitual physical activity in epidemiological studies. Am J Clin Nutr, v.36, p.936-42, 1982.

BRASIL. Casa Civil. Subchefia para Assuntos Jurídicos. Lei de Diretrizes e Bases da Educação Nacional - Lei 9.394 (Lei Ordinária) de 20 de dezembro de 1996.

Disponível em: <http://www.planalto.gov.br/ccivil_03/Leis/L9394.htm>. Acesso em 13 set. 2010.

BRASIL. Ministério da Defesa. Exército Brasileiro. Diretoria de Ensino Preparatório e Assistencial. Regimento Interno dos Colégios Militares (RI/CM) 2010. Disponível em: <http://www.depa.ensino.eb.br/pag_legislacao.htm>. Acesso em: 13 set. 2010.

BRASIL. Ministério da Defesa. Exército Brasileiro. Diretoria de Ensino Preparatório e Assistencial. Regulamento dos Colégios Militares (R-69) - Portaria n. 42, de 06 de fevereiro de 2008. Disponível em: <http://www.depa.ensino.eb.br/Docs/R69_de06Fev08.pdf >. Acesso em: 13 set. 2010.

BRASIL. Ministério da Defesa. Exército Brasileiro. Diretoria de Ensino Preparatório e Assistencial. Sistema Colégio Militar do Brasil, 2009. Disponível em: <http://www.depa.ensino.eb.br/pag_sistemaCM.htm>. Acesso em: 05 set. 2010.

BRASIL. Ministério da Defesa. Exército Brasileiro. Escola de Aperfeiçoamento de Oficiais. Lei de Ensino do Exército - Lei n. 9.786, de 08 de fevereiro de 1999. Disponível em:

<http://www.esao.ensino.eb.br/paginas/secoes/div_ens/spg/legislacao/downloads/lei_en s_mil.pdf >. Acesso em: 13 set. 2010.

BRUM, L. M.; AZAMBUJA, C. R.; REZER, J. F. P.; TEMP, D. S.; CARPILOVSKY, C. K.; LOPES, L. F.; SCHETINGER, M. R. C. Qualidade de vida de professores da área de ciências em escola pública do interior do Rio Grande do Sul. Trab Educ Saúde, v.10, n.1, p.125-45, 2012.

CORBIN, C.; LINDSEY, R. Concepts of physical fitness. $8^{\mathrm{a} e d}$. Dubuque: Brown \& Benchmark Publisher; 1994.

COSTA, R. M.; MILANI, N. S. Perfil do estilo de vida entre professores da rede estadual e particular de ensino regular da cidade de Muriaé, MG. Rev Min Educ Fís, v.15, n.1, p.32-56, 2007.

COUTINHO, R. X.; SANTOS, W. M.; FOLMER, V.; ROCHA, J. B. T.; PUNTEL, R. L. Percepções de professores de ciências, matemática e educação física sobre suas práticas em escolas públicas. Rev Ciênc \& Ideias, v.4, n.1, p.1-18, 2012.

DAMASCENO, V. O.; LIMA, J. R. P.; VIANNA, J. M.; VIANNA, V. R. A.; NOVAES, J. S. Tipo físico ideal e satisfação com a imagem corporal de praticantes de caminhada. Rev Bras Med Esporte, v.11, n.3, p.181-6, 2005. 
DISHMAN, R. K. Determinants of participation in physical activity. In: BOUCHARD, C.; SHEPHARD, R. J.; STEPHENS, T.; SUTTON, J. R.; McPHERSON, B. D. Exercise, fitness and health: A consensus of current knowledge. Illinois: Human Kinetics Books; 1990.

DUDA, J. L. Goal perspectives, participation and persistence in sport. Int J Sport Psychol, v.20, p.42-56, 1989.

FERNANDES, M. H.; GOMES PORTO, G.; DIAS DE ALMEIDA, L. G.; ROCHA, V. M. Estilo de vida de professores universitários: uma estratégia para a promoção da saúde do trabalhador. Rev Bras Prom Saúde; v.22, n.2, p.94-9, 2009.

GANGWISCH, J. E.; MALASPINA, D.; BODEN-ALBALA, B.; HEYMSFIELD, S. B. Inadequate sleep as a risk factor for obesity: analyses of the NHANES I. Sleep, v.28, p.1217-20, 2005.

GASPARINI, S. M.; BARRETO, S. M.; ASSUNÇÃO, A. A. O professor, as condições de trabalho e os efeitos sobre sua saúde. Rev Educ Pesquisa, v.31, n.2, p.189-99, 2005.

GIL, E. L. Adequação do ensino médio do Sistema Colégio Militar do Brasil à Lei de Diretrizes e Bases da Educação Nacional. Dissertação - Escola de Comando e Estado-Maior do Exército, Rio de Janeiro, Mestrado em Educação, 2003, 261 p.

GINZBERG, E. Adolescents at risk conference: overview. J Adolescent Health, v.12, p.588-90, 1991.

GUEDES, D. P.; GUEDES, J. E. R. P.; BARBOSA, D. S.; OLIVEIRA, J. A.; STANGANELLI, L. C. R. Fatores de risco cardiovascular em adolescentes: indicadores biológicos e comportamentais. Arq Bras Cardiologia, v.86, n.6, p.439-50, 2006.

GUIMARÃES, L.; MARTINS, D. A.; GRUBITS, S.; CAETANO, D. Prevalência de transtornos mentais em trabalhadores de uma universidade pública do estado de São Paulo. Rev Bras Saúde Ocup, v.31, n.113, p.7-18, 2006.

KNUTSON, K. L. Sex differences in the association between sleep and body mass index in adolescents. J Pediatr, v.147, p.830-4, 2005.

KOHATSU, N. D.; TSAI, R.; YOUNG, T.; VANGILDER, R.; BURMEISTER, L. F.; STROMQUIST, A. M.; MERCHANT, J. A. Sleep duration and body mass index in a rural population. Arch Intern Med, v.166, p.1701-5, 2006.

MADUREIRA, A. S.; FONSECA, A. S.; MAIA, M. F. M. Estilo de vida e atividade física habitual dos professores de educação física. Rev Bras Cineantropom Desempenho Hum, v. 5, n. 1, p.54-62, 2003.

MATOS, M. G.; SARDINHA, L. B. Estilo de vida activos e qualidade de vida. Lisboa: Exercício e Saúde / Faculdade de Motricidade Humana; 1999. 
MATSUDO, S. M.; MATSUDO, V. R.; ARAÚJO, T.; ANDRADE, D.; ANDRADE, E.; OLIVEIRA, L.; BRAGGION, G. Nível de atividade física da população do estado de São Paulo: análise de acordo com o gênero, idade, nível sócio-econômico, distribuição geográfica e de conhecimento. Rev Bras Ciênc Mov, v.10, n.4, p.41-50, 2002.

MORENO, J. A.; CERVILLÓ, E.; MORENO, R. Importancia de la práctica físicodesportivay del gênero em autoconcepto físico de los 9 a los 23 años. Int J Clin Health Psychol, v.8, n.1, p.171-83, 2008.

PATEL, S. R.; MALHOTRA, A; WHITE, D. P.; GOTTLIEB, D. J.; HU, F. B.

Association between reduced sleep and weight gain in women. Am J Epidemiol, v.164, p.947-54, 2006.

PIÉRON, M. Estilo de vida, prática de atividades físicas e esportivas, qualidade de vida. Fit Perf J, v.3, n.1, p.10-7, 2004.

PITANGA, F. J. G. Epidemiologia, atividade física e saúde. Rev Bras Ciênc Mov, v.10, n.3, p.49-54, 2002.

NAHAS, M. V. Atividade física, saúde e qualidade de vida: conceitos e sugestões para um estilo de vida ativo. Londrina: Midiograf; 2003.

NAHAS, M. V. Revisão de métodos para determinação da atividade física habitual em diversos grupos populacionais. Rev Bras Ativ Fís Saúde, v.1, n.4, p.27-37, 1996.

RICHARDSON, C. R.; AVRIPAS, S. A.; NEAL, D. L.; MARCUS, S. M. Increasing lifestyle physical activity in patients with depression or other serious mental illness. J Psychiatr Practice, v.11, n.6, p.379-88, 2005.

RODRIGUEZ-AÑEZ, C. R.; REIS, R. S.; PETROSKI, E. L. Versão Brasileira do Questionário "Estilo de Vida Fantástico": Tradução e Validação para Adultos Jovens. Arq Bras Cardiol, v.91, n.2, p.102-9, 2008.

SHEPHARD, R. Habitual physical activity and quality of life. Quest, v.48, p.354-65, 1996.

SARDINHA, A.; LEVITAN, M.; LOPES, F.; PERNA, G.; ESQUIVEL, G.; GRIEZ, E.; NARDI, A. Tradução e adaptação transcultural do Questionário de Atividade Física Habitual. Rev Psiquiatr Clínica, v.37, n.1, p.16-22, 2010.

SILVEIRA, E. A.; ARAÚJO, C. L.; GIGANTE, D. P.; BARROS, A.; LIMA, M. S. Validação do peso e altura referidos para o diagnóstico do estado nutricional em uma população de adultos no Sul do Brasil. Cad Saúde Pública, v.21, n.1, p.235-45, 2005.

SOCIEDADE BRASILEIRA DE CARDILOGIA. Cardiômetro: mortes por doenças cardiovasculares no Brasil. Disponível em: <http://www.cardiometro.com.br/anteriores.asp >. Acesso em: 18 out2016.

SUSSER, M.; SUSSER, E. Choising a future for epidemiology. Part I: Eras and paradigms. Am J Public Health, v.86, p.668-73, 1996. 
TELEMA, R.; NUPPONEN, H.; PIÉRON, M. Physical activity among young people in the context of lifestyle. Eur Phys Educ Rev, v.11, n.2, p.115-37, 2005.

TOLEDO, R. F. Educação, Saúde e Meio Ambiente: uma pesquisa-ação no Distrito de Iauaretê do Município de São Gabriel da Cachoeira/AM., 2006, 326f. Tese [Doutorado] - Faculdade de Saúde Pública, Universidade de São Paulo; 2006.

VALOIS, R. F.; UMSTATTD, M. R.; ZULLIG, K. J.; PAXTON, R. J. Physical activity behaviors and emotional self-efficacy: Is there a relationship for adolescents? J Sch Health, v.78, n.6, p.321-327, 2008.

VEDOVATO, T. G.; MONTEIRO, M. I. Perfil sociodemográfico e condições de saúde e trabalho dos professores de nove escolas estaduais paulistas. Rev Esc Enferm USP, v.42, n.2, p.290-97, 2008.

\section{VIEIRA, H. P. Estresse ocupacional, síndrome de Burnout e Hardiness em} professor de Colégio Militar. Dissertação - Universidade Católica Dom Bosco, Campo Grande, Mestrado em Psicologia, 2007, 121p. Disponível em:

$<$ http://site.ucdb.br/public/md-dissertacoes/8018-estresse-ocupacional-sindrome-deburnout-e-hardiness-em-professores-de-colegio-militar.pdf >. Acesso em: 22 fev. 2013.

WORLD HEALT ORGANIZATION (WHO). BMI classification. Estados Unidos; 2006. Disponível em: <http://apps.who.int/bmi/index.jsp?introPage=intro_3.html>. Acesso em: 12 jul. 2012.

WORLD HEALT ORGANIZATION (WHO). The World Health Report 1998 - Life in the $21^{\text {st }}$ Century: a vision for all. Geneva; 1998.

\section{Como referenciar este artigo}

AZAMBUJA, Cati Reckelberg et al. Estudio de caso sobre la integración socioeducativa de familias inmigrantes en Granada (España). Revista Ibero-Americana de Estudos em Educação, Araraquara/SP, v. 11, n. 3, p.1519-1538, 2016. Disponível em: <https://dx.doi.org/10.21723/riaee.v11.n3.6239>. E-ISSN: 1982-5587.

Submetido em: 06/09/2013

Aprovação final em: 04/07/2016 